\title{
Statin-Associated Necrotizing Myopathy: A Feared Complication
}

\author{
Suong Nguyen ${ }^{1}$, Swetha Ann Alexander ${ }^{1}$, Sandra Apenteng ${ }^{1}$, Andrew Castiglione ${ }^{2}$ \\ 1. Internal Medicine, University of Connecticut, Farmington, USA 2. Internal Medicine, Hartford Hospital, Hartford, \\ USA
}

Corresponding author: Suong Nguyen, sunguyen@uchc.edu

\begin{abstract}
Statins are a group of frequently-prescribed drugs with proven cardiovascular risk-benefit. The most common adverse effects include weakness and myalgias. However, prescribers need to be aware of a less common complication, statin-associated necrotizing myopathy, which can occur at any time during the treatment course and has been found to be $<0.1 \%$ of adverse effects. High suspicion is warranted when patients taking statins develop weakness and myalgia. Increased risk of muscle injury has been observed when using gemfibrozil in combination with statins and should be avoided. We present a case of an elderly male with chronic use of combination lipid-lowering agents who initially presented with proximal weakness. He was diagnosed with statin-associated necrotizing myopathy and subsequently developed rapid end-stage renal disease in the setting of severe rhabdomyolysis. The case report discusses the work-up of proximal muscle weakness with focus on the importance of early recognition and prompt management of rhabdomyolysis to avoid life-threatening complications.
\end{abstract}

Categories: Internal Medicine, Neurology, Rheumatology

Keywords: statin, statin-induced, myopathy, rhabdomyolysis, weakness, hmg-coa reductase

\section{Introduction}

Since the 1980s, statins (hydroxymethylglutaryl-CoA reductase inhibitors) have undergone remarkable changes from semi-synthetic to synthetic formulations, with atorvastatin being the most commonly prescribed [1-3]. Large clinical trials have proven primary and secondary prevention of cardiovascular events contributing to its widespread use and integration into clinical practice guidelines [4]. Among the very same cohorts that showed significant cardiovascular benefits, adverse effects ranging from myalgia to necrotic myopathy have been reported. Statin-induced rhabdomyolysis occurs in 0.44 per 10,000 patients a year, with incidence increasing to 5.98 per 10,000 patients when combined with fibrates [3,5].

We describe a case of an elderly man presenting with proximal limb weakness diagnosed with statinassociated necrotizing myopathy in the setting of decompensated renal function. We aim to discuss the workup of proximal muscle weakness and current treatment regimens for severe cases of rhabdomyolysis.

Review began 09/10/2020 Review ended 11/24/2020 Published 11/24/2020

(c) Copyright 2020 Nguyen et al. This is an open access article distributed under the terms of the Creative Commons Attribution License CC-BY 4.0., which permits unrestricted use, distribution, and reproduction in any medium, provided the original author and source are credited.

\section{Case Presentation}

A 74-year-old male with a history of atrial fibrillation, heart failure with reduced ejection fraction (HFrEF), hypertension, hyperlipidemia, insulin-dependent diabetes, active diverticulitis on antibiotics, and chronic kidney disease (CKD) stage III (baseline S.cr 2-2.5 mg/dL) presented with two weeks of worsening fatigue and weakness with a $20 \mathrm{lb}$ weight loss. He was hemodynamically stable, alert and oriented, saturating at $92 \%$ on room air with normal lung sounds. Symmetrical proximal muscle weakness of the bilateral upper and lower extremities was present with failure to resist force at hip flexors. Distal strength, sensation, and reflexes were intact and symmetric bilaterally and no rashes were noted. Metabolic profile indicated anion gap metabolic acidosis and acute kidney injury with serum creatinine of $5.3 \mathrm{mg} / \mathrm{dL}$, blood urea nitrogen (BUN) of $78 \mathrm{mg} / \mathrm{dL}$, bicarbonate of $15 \mathrm{mmol} / \mathrm{L}$ and normokalemia with lactic acid of $1.2 \mathrm{mmol} / \mathrm{L}$, creatinine kinase (CK) levels of 9,951 U/L (24-204 U/L) and urinalysis with proteinuria and myoglobinuria. Medication reconciliation revealed current intake of rosuvastatin $40 \mathrm{mg}$ daily (previously on high-dose atorvastatin) and gemfibrozil $600 \mathrm{mg}$ twice daily.

MRI revealed diffuse and patchy muscular edema of the bilateral thighs, seen in Figure 1. Further work-up demonstrated thyroid-stimulating hormone (TSH) of $1.10 \mathrm{mIU} / \mathrm{L}$, c-reactive protein (CRP) of $1.55 \mathrm{U} / \mathrm{L}$, erythrocyte sedimentation rate (ESR) of $114 \mathrm{MM} / \mathrm{HR}$, antinuclear antibody (ANA) of 1:80, complement total (CH50) of $>60$ units/mL (31-60 U/mL), 3-hydroxy-3-methyl-glutaryl-coenzyme A reductase (HGMR) antibody, immunoglobulin $\mathrm{G}(\mathrm{IgG})<3$ units $(0-19$ units) and negative myositis panel. 


\section{Cureus}

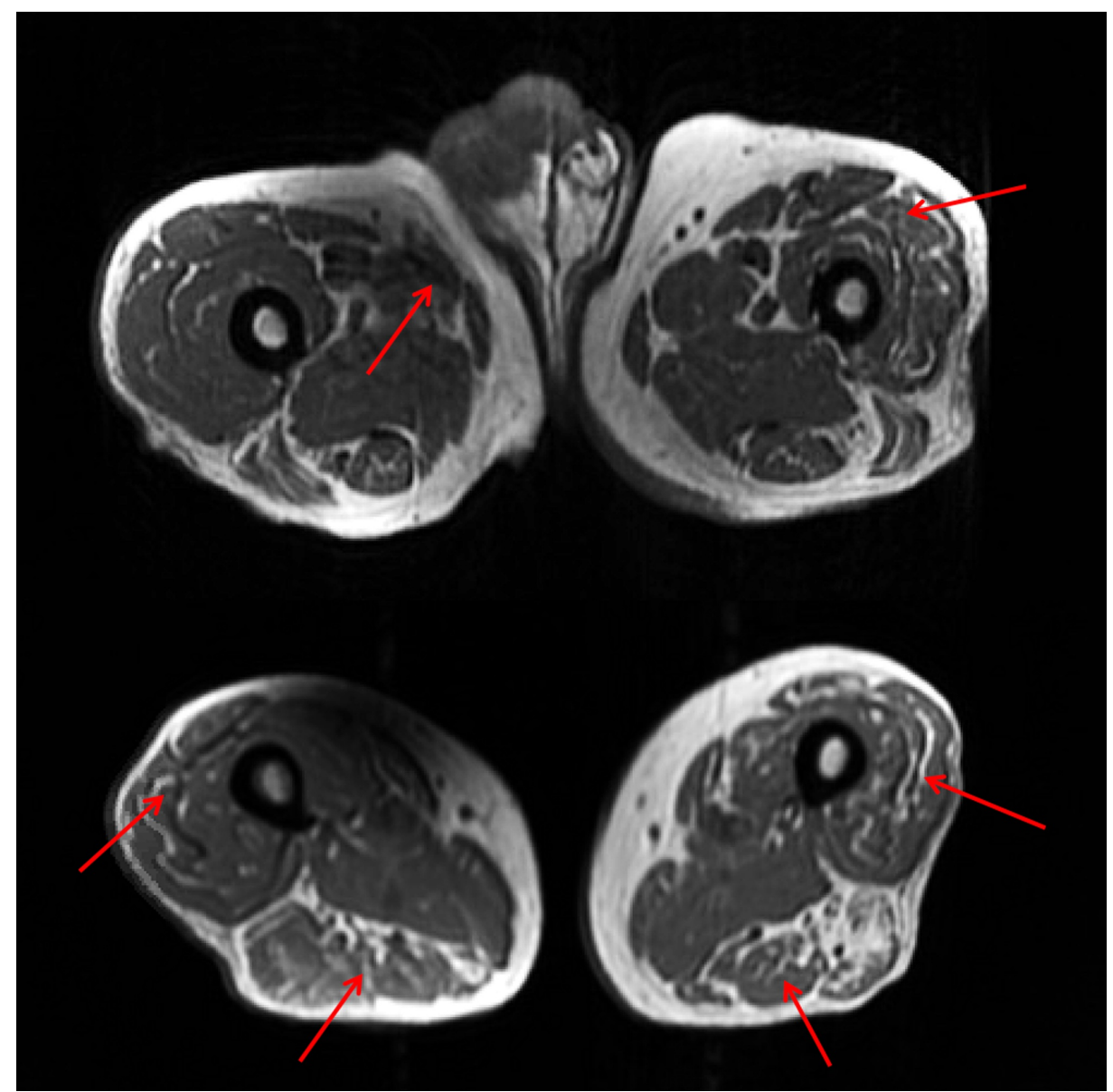

FIGURE 1: Axial T1 fast spin-echo (FSE) of the upper and lower bilateral thighs with mild edema diffusely in the anterior muscles of the proximal left and right thigh (top) with increased hyperintensity in the mid to distal thigh muscles bilaterally suggestive of muscular edema (bottom).

Despite statin discontinuation and aggressive intravenous hydration with lactated ringers, his symptoms progressed and CK levels continued to rise. Nephrology and Rheumatology services were consulted within 24 hours of admission for recommendations given high suspicion for statin-induced necrotizing myopathy. He was subsequently started on methylprednisone $60 \mathrm{mg}$ twice daily and intravenous immunoglobulin (IVIG) within the same day. On day seven of admission, the patient became acutely encephalopathic in the setting of uremia and he was started on continuous renal replacement therapy for worsening anuric acute renal failure with acute tubular necrosis. Electromyography (EMG) was deferred due to severity of the course and left thigh muscle biopsy was performed following immunosuppressive treatment on day 16 , which revealed marked myofiber necrosis and regeneration consistent with acute rhabdomyolysis. Unfortunately, the patient continued to deteriorate and suffered severe depression leading to decline of further treatment resulting in transition to comfort care on day 19 of admission.

\section{Discussion}

Before diagnosing a patient with statin-induced myopathy it is important to evaluate differential diagnoses with similar presentations. Proximal weakness is defined in terms of function. Patients with proximal weakness often face new onset difficulty performing tasks such as climbing stairs or combing hair. Given various causes of proximal weakness (Table 1) it is pertinent to obtain a thorough medical history, medication reconciliation, alcohol and drug history, and evaluation for toxins and metabolic disturbances. 


\section{Cureus}

\section{Diagnosis}

Inflammatory

Polymyositis

Dermatomyositis

Skeletal muscle vasculitis with chronic rheumatological conditions

Endocrine

Hypothyroidism/hyperthyroidism

Cushing's syndrome

Electrolyte disorders

Hypokalemia, hypophosphatemia, hypocalcemia, hypo/hypernatremia

Metabolic myopathy

Carbohydrate, lipid or purine metabolic abnormality

Drugs and toxins

Alcohol

Cocaine and heroine

HMG-CoA reductase inhibitors (Otherspenicillamine, zidovudine, colchicine, antimalarial drugs)

Rhabdomyolysis

Crush trauma, seizures, delirium tremens, extreme exertion, malignant hyperthermia

Inherited myopathies

Muscular dystrophy
Characteristic Features

Proximal muscle weakness with elevated CK and aldolase. EMG shows typical features of muscle involvement. Biopsy reveals cellular infiltrate within the fascicle with cytotoxic CD8+ T cells.

Features similar to polymyositis with specific skin findings of Gottron's papules or heliotrope rash. Biopsy reveals cellular infiltrate mainly in perifascicular area with Blymphocytes.

Associated with a chronic inflammatory condition and may have systemic symptoms. Biopsy reveals fibrinoid necrosis of the vessel wall with transmural inflammation.

Proximal muscle weakness with myoedema and abnormal thyroid-stimulating hormone levels with resolution occurring when euthyroid.

Associated with proximal myalgias with normal EMG and CK and myoglobin levels. Biopsy reveals atrophy of muscles.

Acute to subacute onset with cramp-like weakness with normal to elevated CK levels. EMG may show "myopathic" motor unit potentials with biopsy revealing myofiber vacuolation and necrosis.

Onset may be at young-age with exercise intolerance and cramping of proximal and distal muscles. Systemic involvement may present causing cardiomyopathy and neuropathy.

May present with acute or chronic rhabdomyolysis with biopsy revealing diffuse muscle atrophy.

May lead to non-traumatic rhabdomyolysis.

Persistently elevated CK levels with muscle histology revealing muscle necrosis with minimal inflammation.

Markedly elevated CK levels with myalgia and myoglobinuria.

Elevated CK and transaminases at onset of birth and may further require genetic analysis.

\section{TABLE 1: Differential diagnosis of proximal weakness and characteristic features}

CK: creatinine kinase, EMG: electromyography, HMG-CoA: 3-hydroxy-3-methylglutaryl coenzyme A

Myopathy is a broad term used to describe muscular dysfunction with skeletal muscle weakness being a principal symptom. Indirect and direct muscle injury may lead to myopathies and in its most severe instances may present as rhabdomyolysis. Predisposing risk-factors include elderly age, female gender, renal insufficiency, hepatic dysfunction, hypothyroidism and polypharmacy. As mentioned prior, statins have been shown in numerous large cohort studies to increase the risk of rhabdomyolysis with an estimated 2.3 per 10,000 person-years [5]. Although all statins increase the risk of muscle injury by $0.1 \%$ to $0.5 \%$, rosuvastatin at varying doses has been shown in studies to increase the risk of muscle involvement to $1 \%$ compared to other statins [5,6]. A small retrospective study involving 45 patients observed the mean duration of statin therapy of 6.3 months before onset of symptoms with a mean duration of myalgia of 2.3 
months after statin discontinuation [7]. An increased risk of myopathy has also been observed between drug-drug interactions, particularly with fibrates, with reports of a 5.5-fold increase with statin-fibrate therapy compared to statin monotherapy through a mechanism that increases the overall plasma concentration of the used statin [5,7]. In doing so, 3-hydroxy-3-methylglutaryl coenzyme A (HMG-CoA) reductase becomes inhibited preventing conversion to mevalonate, a precursor for producing isoprenoids $[3,8]$. The lack of isoprenoids causes inactivation of GTPases preventing cell membrane anchorage altering cellular function causing decreased cholesterol concentrations in the cell membrane with subsequently impaired mitochondrial enzyme activity leading to muscle injury [3].

Rhabdomyolysis should be suspected in patients presenting with acute weakness, myalgias, acute renal failure, and myoglobinuria following investigation starting with routine lab work. In severe cases, a basic metabolic profile (BMP) may reveal acute renal dysfunction with metabolic acidosis secondary to lactic acidosis with electrolyte abnormalities such as hypocalcemia, hyperkalemia, and hyperphosphatemia $[2,5,9]$. Myonecrosis leads to protease release into the systemic circulation causing elevations of myoglobin, CK, lactate dehydrogenase, and hydroxybutyrate dehydrogenase, which lead to elevated transaminases reflecting hepatic dysfunction seen in up to $25 \%$ of patients with rhabdomyolysis [10]. Serum CK levels may be greater than 10 times the upper limit of normal with rise in two to 12 hours after onset of injury peaking at three to five days and declining in six to 10 days [3,7,9,11]. Urinalysis may reveal "tea colored urine" due to myoglobinuria. It is imperative to perform a medical reconciliation and drug toxicity screen to exclude illicit substances as potential contributors [9]. It is necessary to rule out autoimmune causes of myopathy by testing for myositis specific antibodies (MSA) including, but not limited to, anti-Sjögren's type A (anti-Jo1/SSA), anti-Sjögren's type B (anti-La/SSB), and anti-signal recognition particle (anti-SRP) for polymyositis, dermatomyositis, systemic lupus erythematosus, and Sjögren's syndrome [8,11]. In certain circumstances in which immunosuppressives are promptly started before MSA results are available, it may necessitate muscle biopsy to confirm the presence of perivascular atrophy and/or lymphocytic cellular infiltrates related to these autoimmune disorders [11]. Further testing for 3-hydroxy-3-methylglutaryl coenzyme A reductase (HGMR) antibody will evaluate for statin necrotizing myopathy and thyroid stimulating hormone (TSH) will determine presence of thyroid dysfunction.

In situations of worsening rhabdomyolysis further diagnostic studies are warranted. An MRI can be used to assess the extent of muscle involvement and determine the site of muscle biopsy, which is typically the thigh or biceps. In statin-associated myopathy, MRI demonstrates the presence of intramuscular fatty infiltration with or without edema with minimal inflammation [11,12]. Electromyography generally presents irritable myopathy and muscle biopsy reveals myofiber atrophy with necrosis without inflammation [12].

Statins and offending agents should be discontinued immediately when symptoms present. Treatment begins with volume resuscitation to expand the extracellular fluid compartment. Aggressive IV hydration should be administered early and continued until plasma CK levels decrease to 1,000 U/1 or below $[5,6]$. Sodium bicarbonate may be used to alkalinize urine to decrease cast formation and minimize renal tubule injury secondary to the toxic effects of myoglobin. Due to lack of studies, there are no guidelines recommending fluid type and rate in regard to hydration in the setting of rhabdomyolysis.

Steroids, intravenous immunoglobulin (IVIG), and immunomodulating agents have become the mainstay treatment for statin-associated myopathy observed in the literature [8,12]. If symptoms continue to worsen despite statin discontinuation, then prompt initiation of steroids, often at the gestalt of the clinician providing care, is warranted. The use of prednisone $1-2 \mathrm{mg} / \mathrm{kg} / \mathrm{day}$ dosage or pulse dose steroids of methylprednisone $1 \mathrm{~g}$ daily for three days in cases has been and has demonstrated a positive effect $[8,12]$. With failed clinical improvement in weeks to months with steroid administration, studies have demonstrated some efficacy of using immunomodulating agents such as methotrexate or rituximab as second line agents $[8,12]$. Further management with IVIG monotherapy is considered with doses of $2 \mathrm{mg} / \mathrm{kg}$ divided over a two- to five-day course. IVIG has shown modest success in two different studies demonstrating at least a partial improvement in patient's symptoms by $36 \%$ and $54 \%$, with complete to near-complete resolution of symptoms occurring $64 \%$ and $46 \%$ respectively $[13,14]$. It is unfortunate we will not know if our patient would have had resolution of his symptoms if treatment were to have continued. There is also a question if further immunomodulating agents would have had any effect on his illness.

\section{Conclusions}

Statins are commonly prescribed medications in medical practice and should be discontinued with the first signs of statin intolerance. In patients presenting with proximal muscle weakness; inflammatory, infectious, toxic, endocrine, or metabolic causes should be thoroughly evaluated. Statin-associated necrotic myopathy may occur at any time after drug initiation and should be considered in the patient with muscle weakness irrespective of the time course of initiation and if left unconsidered may be fatal. Prompt and aggressive volume restoration is critical to prevent progression to acute renal failure and the need for renal replacement therapy. Steroids and immunomodulating treatment should be considered if symptoms fail to improve. However, there continues to be a need for randomized controlled trials to further study the treatment of statin-associated necrotic myopathy as patients continue to be treated based on symptom severity and clinical presentation relying on observations in retrospective studies and expert consensus. 


\section{Additional Information \\ Disclosures}

Human subjects: Consent was obtained by all participants in this study. Conflicts of interest: In compliance with the ICMJE uniform disclosure form, all authors declare the following: Payment/services info: All authors have declared that no financial support was received from any organization for the submitted work. Financial relationships: All authors have declared that they have no financial relationships at present or within the previous three years with any organizations that might have an interest in the submitted work. Other relationships: All authors have declared that there are no other relationships or activities that could appear to have influenced the submitted work.

\section{References}

1. Arnett DK, Blumenthal RS, Albert MA, et al.: 2019 ACC/AHA guideline on the primary prevention of cardiovascular disease: Executive summary: A report of the American College of Cardiology/American Heart Association task force on clinical practice guidelines. Circulation. 2019, 140:10.1161/cir.0000000000000678

2. Gabow PA, Kaehny WD, Kelleher SP: The spectrum of rhabdomyolysis . Medicine. 1982, 61:141-152. 10.1097/00005792-198205000-00002

3. Sakamoto K, Kimura J: Mechanism of statin-induced rhabdomyolysis. J Pharmacol Sci. 2013, 123:289-294. 10.1254/jphs.13r06cp

4. Endo A: A historical perspective on the discovery of statins . Proc Jpn Acad Ser B, Phys Biol Sci. 2010, 86:484-493. 10.2183/pjab.86.484

5. Graham DJ, Staffa J, Shatin D, et al.: Incidence of hospitalized rhabdomyolysis in patients treated with lipid-lowering drugs. JAMA. 2004, 292:2585-2590. 10.1001/jama.292.21.2585

6. Rosuvastatin NDA 21-366 N000 Resubmission Amendment . (2003). Accessed: May 21, 2020: https://www.accessdata.fda.gov/drugsatfda_docs/nda/2003/21-366_Crestor_Medr_P1.pdf.

7. Hansen KE, Hildebrand JP, Ferguson EE, Stein JH: Outcomes in 45 patients with statin-associated myopathy. Arch Intern Med. 2005, 165:2671-2676. 10.1001/archinte.165.22.2671

8. Sweidan AJ, Leung A, Kaiser CJ, Strube SJ, Dokukin AN, Romansky S, Farjami S: A case of statin-associated autoimmune myopathy. Clin Med Case Rep. 2017, 10: 10.1177/1179547616688231

9. Newman CB, Preiss D, Tobert JA, et al.: Statin safety and associated adverse events: a scientific statement from the American Heart Association. Arterioscl Throm Vas. 2019, 39:38-81.

10.1161/atv.0000000000000073

10. Akmal M, Massry SG: Reversible hepatic dysfunction associated with rhabdomyolysis . Am J Nephrol. 1990, 10:49-52. 10.1159/000168053

11. Nance JR, Mammen AL: Diagnostic evaluation of rhabdomyolysis. Muscle Nerve. 2015, 51:793-810. 10.1002/mus.24606

12. Villa L, Lerario A, Calloni S, et al.: Immune-mediated necrotizing myopathy due to statins exposure. Acta Myol. 2018, 37:257-262.

13. Mohassel P, Mammen AL: Statin-associated autoimmune myopathy and anti-HMGCR autoantibodies . Muscle Nerve. 2013, 48:477-483. 10.1002/mus.23854

14. Grable-Esposito P, Katzberg HD, Greenberg SA, Srinivasan J, Katz J, Amato AA: Immune-mediated necrotizing myopathy associated with statins. Muscle Nerve. 2009, 41:185-190. 10.1002/mus.21486 